\title{
Hyaluronidase inhibits reactive adipogenesis and inflammation of colon and skin
}

Tatsuya Dokoshi, ${ }^{1}$ Ling-juan Zhang, ${ }^{1}$ Teruaki Nakatsuji, ${ }^{1}$ Christopher A. Adase, ${ }^{1}$ James A. Sanford, ${ }^{1}$ Rudolph D. Paladini, ${ }^{2}$ Hiroki Tanaka, ${ }^{3}$ Mikihiro Fujiya, ${ }^{4}$ and Richard L. Gallo ${ }^{1}$

'Department of Dermatology, UCSD, La Jolla, California, USA. ${ }^{2}$ Halozyme Therapeutics Inc., San Diego, California, USA. ${ }^{3}$ Department of Legal Medicine, Asahikawa Medical University, Asahikawa, Japan. ${ }^{4}$ Division of Gastroenterology and Hematology/Oncology, Department of Medicine, Asahikawa Medical University, Asahikawa, Japan.

In this study we evaluated the role of hyaluronan (HA) in reactive adipogenesis, a local expansion of preadipocytes that provides host defense by release of antimicrobial peptides. We observed that HA accumulated during maturation of adipocytes in vitro and was associated with increased expression of preadipocyte factor 1 , zinc finger protein 423 , and early $B$ cell factor 1 . Although $\mathrm{HA}$ is normally abundant in the extracellular matrix, a further increase in HA staining occurred in mice at sites of reactive adipogenesis following injury of colon by dextran sodium sulfate or injury of skin from infection with Staphylococcus aureus. HA also abundantly accumulated around adipocytes seen in the colons of patients with inflammatory bowel disease. This HA was necessary for adipocyte maturation because digestion of HA by administration of soluble hyaluronidase or transgenic expression of hyaluronidase 1 inhibited adipogenesis in vitro and in vivo. Furthermore, hyaluronidase also suppressed inflammation of both skin and colon and decreased antimicrobial peptide expression by developing preadipocytes. This resulted in increased bacterial transit across the epithelial barrier despite decreased tissue injury from inflammation. These observations suggest HA plays an important role in reactive adipogenesis and host defense after injury.

Conflict of interest: RDP is an employee of Halozyme Therapeutics Inc. RLG is a consultant for and has equity interest in Senté and MatriSys Bioscience.

License: Copyright 2018, American Society for Clinical Investigation.

Submitted: June 21, 2018 Accepted: September 13, 2018 Published: November 2, 2018

\section{Reference information:} JCI Insight. 2018;3(21):e123072. https://doi.org/10.1172/jici. insight.123072.

\section{Introduction}

Hyaluronan (HA) is a glycosaminoglycan that is widely distributed in the extracellular matrix of many tissues (1-3). It is synthesized at the cell surface as a single long-chain carbohydrate of extremely high molecular weight (HMW-HA) that serves physiological roles in macromolecular filtering, water homeostasis, lubrication, and cell adhesion and migration (4). Furthermore, upon tissue injury HMW-HA is rapidly digested to smaller, low-molecular-weight fragments (LMW-HA) that have diverse biological functions, including acting as an endogenous signal of epithelial tissue damage (5). The synthesis and catabolism of HA have been shown to be important to promote epithelial renewal, enhance tight junction formation in epithelia, influence tissue fibrosis, and regulate dendritic cell maturation in many organ systems, including lungs, gut, and skin (6-11).

In addition to its roles in immune regulation, HMW-HA has been reported to play a role in the control of fat accumulation within mice fed a high-fat diet (12). This function of HA during adipogenesis could affect the control of adipose development during morphogenesis and tissue injury because a local increase in adipogenesis occurs in the dermis during wound repair and hair morphogenesis (13-15) and at other sites of epithelial injury, including IBD. The accumulation of mesenteric fat in the colon, also known as "creeping fat," and the appearance of the "fat halo sign" within the submucosa of the small intestine, are well-known signs associated with inflammation in Crohn's disease and idiopathic $\operatorname{IBD}(3,8,9)$. The functional significance of the accumulation of fat in IBD is not well understood.

We recently observed that a local increase of dermal adipocytes occurs in response to bacterial infection and have named this process "reactive adipogenesis" due to the acute and localized reaction and role in production of antimicrobial peptides to inhibit infection (16). The present study sought to evaluate whether HA might influence adipogenesis and elucidate the potential role of HA and adipogenesis during the injury response of the colon and skin. Our results show digestion of HA inhibited this adipogenesis, 
inflammation, and resistance to microbial penetration of both skin and intestine. These observations link HA catabolism to the reactive adipogenesis response and provide evidence suggesting that the regulation of HA accumulation could be a therapeutically useful antiinflammatory strategy.

\section{Results}

In vitro adipogenesis is inhibited by hyaluronidase. To understand the potential role of HA to enable preadipocytes to differentiate to mature adipocytes, HA was first studied in an in vitro tissue culture system. A large increase in HA was measured in the culture supernatant of the mouse preadipocyte cell line (3T3-L1) during differentiation to mature adipocytes (Figure 1A). This HA was high molecular weight and could be effectively digested by addition of PEGPH20 (Figure 1B). Digestion of HMW-HA also decreased production of lipids (Figure 1, C and D) and decreased expression of the adipocyte differentiation markers adiponectin (Adipoq), preadipocyte factor 1 (Pref1), and CEBP- $\alpha$ (Cebp $\alpha$ ). An increase in cell proliferation and BrdU incorporation, as well as a decrease in expression of early B cell factor 1 (Ebf1), Adipoq, and zinc finger protein 423 (Zfp521), were also seen following HA digestion (Figure 1, E-G, and Supplemental Figure 1, A-E; supplemental material available online with this article; https://doi.org/10.1172/jci. insight.123072DS1). These observations were all consistent with a decrease in differentiation to mature adipocytes that was also seen following HA digestion (Supplemental Figure 1, A and B). Furthermore, and consistent with the role of early adipogenesis in innate antimicrobial defense, gene expression for the antimicrobial peptide cathelicidin (Camp) was decreased with the addition of hyaluronidase (Figure $1 \mathrm{H}$ ). Taken together, these results all suggested that digestion of HMW-HA with hyaluronidase inhibits adipocyte differentiation.

An increase in adipocytes and HA occurs in colitis. Skin infection results in local reactive adipogenesis (16). To determine whether an increase in adipocytes results from tissue injury to another epithelial surface, acute colitis was induced in mice through oral administration of $3 \%$ dextran sulfate (DSS). Increased accumulation of mesenteric fat was observed 7 days after induction of colitis by DSS (Figure 2A). Histological evaluation revealed prominent thickening of the submucosal layer, inflammation, epithelial disruption, and accumulation of mature adipocytes at day 42 after repeated DSS administration (Figure 2B). Sequencing of RNA extracted from the submucosa identified an increase in 608 genes and a decrease of 154 genes altered during colitis (Figure 2C and Supplemental Table 1), and gene ontology analysis revealed a prominent increase in genes related to lipid localization and inflammation (Figure 2D). Quantitative PCR (qPCR) validated that DSS colitis was associated with a significant increase in the expression of genes associated with adipogenesis, including Pref1, Zfp 423, and Ebf1 (Figure 1E; refs. 17-19). Activation of Zfp 423 during colitis was confirmed in $Z f p 423^{\mathrm{LacZ}}$ reporter mice that showed a large increase in $\beta$-gal staining of the colon, of surrounding fat (Figure 2F), and of expansion in the submucosal layer (Figure 2G). Immunohistochemistry confirmed that protein expression of PREF1 occurred within cells in the thickened submucosal layer (Supplemental Figure 2). Similar to these observations during DSS colitis in mice, an increase in the expression of Pref1 was also observed in human tissues, with prominent staining of Pref1 observed in cells in the submucosal layer of involved regions from subjects with Crohn's disease and ulcerative colitis (Figure 2, H and I, and Supplemental Figure 3). These observations demonstrate that reactive adipogenesis can be induced in mouse DSS colitis and occurs in human colitis.

We next examined whether an increase in HA could be observed associated with the increase in adipocytes seen during colitis. Similar to the increase in HMW-HA that was seen during adipogenesis in vitro, HA accumulated in adipose tissue seen in sections from human colitis and in the murine experimental colitis system (Figure 3, A-C). Because digestion of HMW-HA was observed to inhibit adipocyte development in vitro, we hypothesized that digestion of HA in vivo could provide a previously unexplored method to influence reactive adipogenesis in inflamed tissues.

Digestion of $H A$ inhibits reactive adipogenesis. To evaluate whether reactive adipogenesis could be influenced by HA digestion, we used 2 independent methods for in vivo delivery of hyaluronidase: a transgenic mouse model that permits early embryonic expression of human hyaluronidase 1 (Ella/Hyal1) (7) and a pharmacological model of HA digestion by injection of PEGPH20. These 2 methods for delivery of hyaluronidase were then evaluated in 2 models of reactive adipogenesis: skin infection and DSS colitis. These methods effectively digested HA in the skin and colon (Supplemental Figure 4).

The expression of Hyal-1 or the administration of PEGPH20 was each associated with a suppression of expansion of dermal white adipose tissue (DWAT) following injury by Staphylococcus aureus as seen by 
A

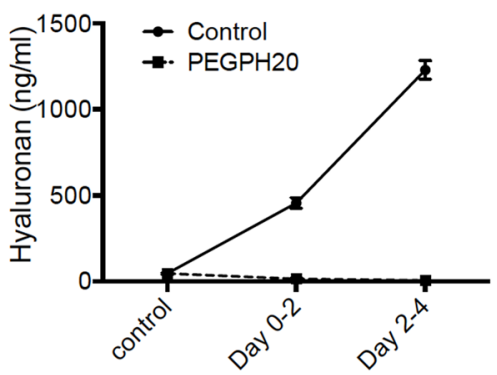

B

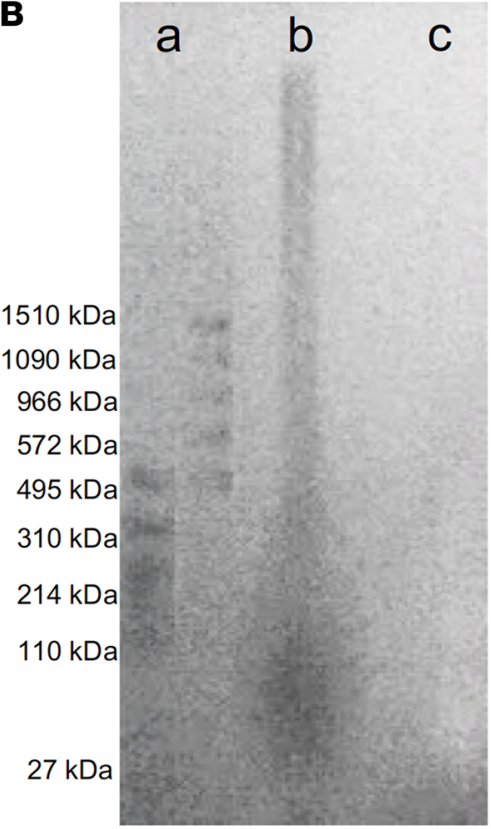

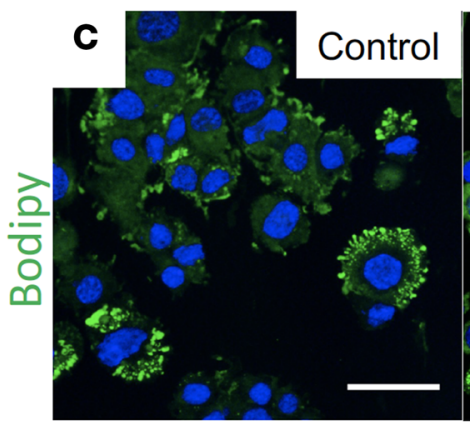
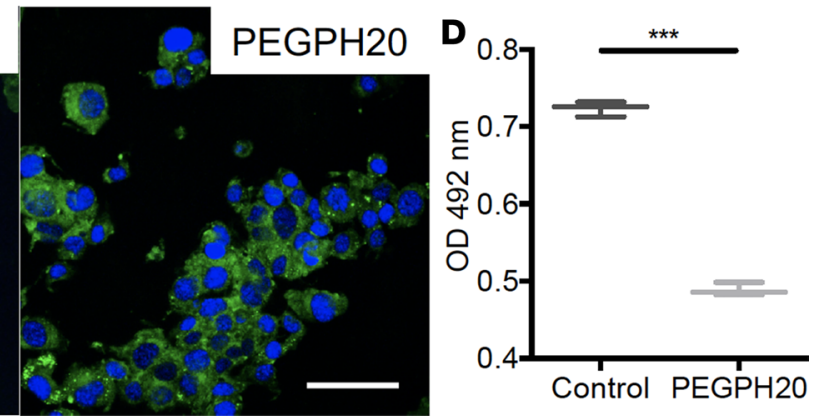

E

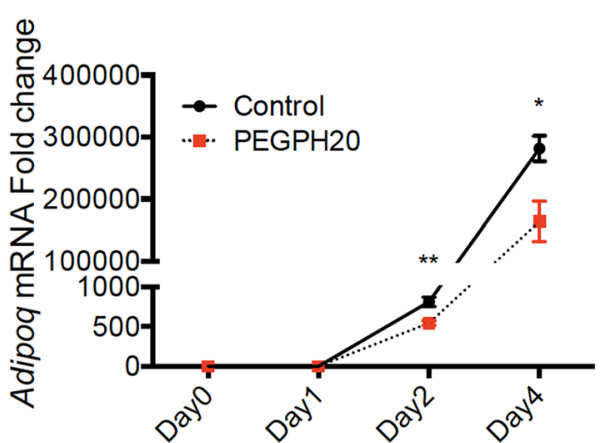

$\mathbf{F}$

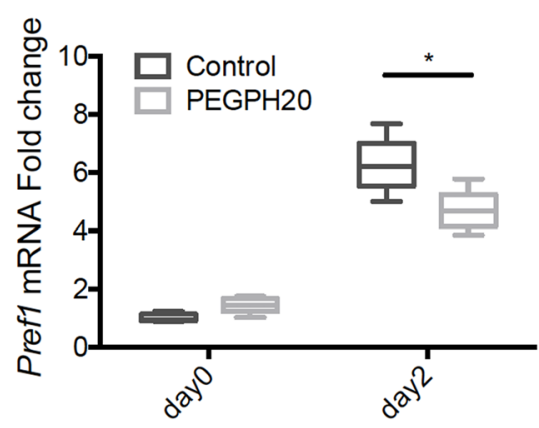

G

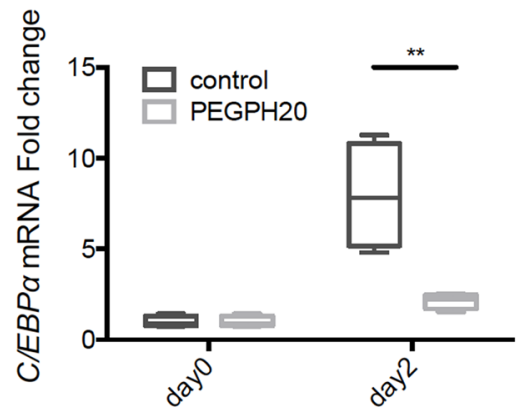

H

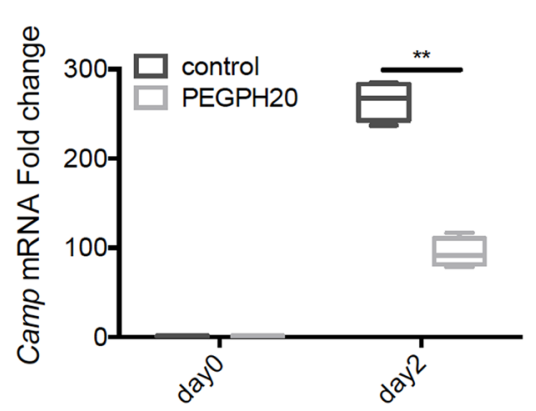

Figure 1. Hyaluronidase inhibits in vitro adipogenesis. Mouse preadipocytes (3T3-L1) were differentiated by the addition of adipocyte differentiation media (see Methods) with or without the addition of $20 \mu \mathrm{g} / \mathrm{ml}$ of PEGylated recombinant human hyaluronidase PH20 (PEGPH20). (A) HA concentrations in 3T3-L1 supernatant over time after addition of differentiation media with and without PEGPH2O $(n=6)$. (B) Size distribution of HA by gel electrophoresis. HA was extracted from equal volumes of supernatant collected at day 4: a: molecular weight marker, b: control, and c: PEGPH20. (C) Lipid staining of 3T3-L1 cells using boron dipyrromethene (BODIPY) on day 2 of differentiation with or without PEGPH20 $(n=5)$. Scale bar: 50 microns. (D) Total lipid abundance measured by Oil Red 0 staining at OD $492 \mathrm{~nm}$ cells at day 5 of differentiation with or without PEGPH20 $(n=3)$. (E) Expression of Adipoq mRNA under culture conditions identical to (A). (F and G) Relative expression of mRNA for Pref1, C/EBP $\alpha$, and Camp during differentiation. Data in $\mathbf{D}$ and $\mathbf{F}$-H are represented using box-and-whisker plots, with boxes representing the interquartile range (IQR), lines representing the median value, and whiskers representing minimum and maximum values, whereas data in $\mathbf{E}$ is represented as mean $\pm \mathrm{SEM}$; ${ }^{*} P<0.05,{ }^{* *} P<0.01$ (Student's $t$ test), and ${ }^{* * *} P<0.001$ (Student's $t$ test).

decreased expansion of the adipocyte layer, lipid droplet staining in the dermis, and lower expression of Cebp $\alpha$ (Figure 4, A-C). Similarly, both methods of hyaluronidase treatment also inhibited the expansion of adipocytes in the colon damaged by DSS. Hyaluronidase inhibited PREF1 staining induced by DSS (Figure 4D); decreased the population of steady state and developing preadipocytes in the mesentery of the colon as defined by CD31 negative, CD45 negative, PDGFR- $\alpha$ positive, and spinocerebellar ataxia type 1 (SCA1) positive (Figure 4, E and F, and ref. 17); and inhibited the increase in Pref1 mRNA (Figure 4G).

Having observed an association between hyaluronidase treatment and an inhibition of adipocyte development, we compared this effect to another method frequently used to inhibit adipogenesis: use of bisphenol A diglycidyl ether (BADGE), a PPAR $\gamma$ agonist. Measurement of Pref 1 mRNA expression in the colon before and after DSS colitis showed that BADGE had a similar effect to digestion of HA. Taken together, these observations support the conclusion that hyaluronidase can inhibit adipogenesis of both skin and colon.

Hyaluronidase treatment inhibits tissue inflammation. The production of adipokines and antimicrobial peptides by adipocytes has been hypothesized to be important to both promote inflammation and protect against infection $(16,20)$. Therefore, given observations that hyaluronidase can inhibit adipogenesis and 

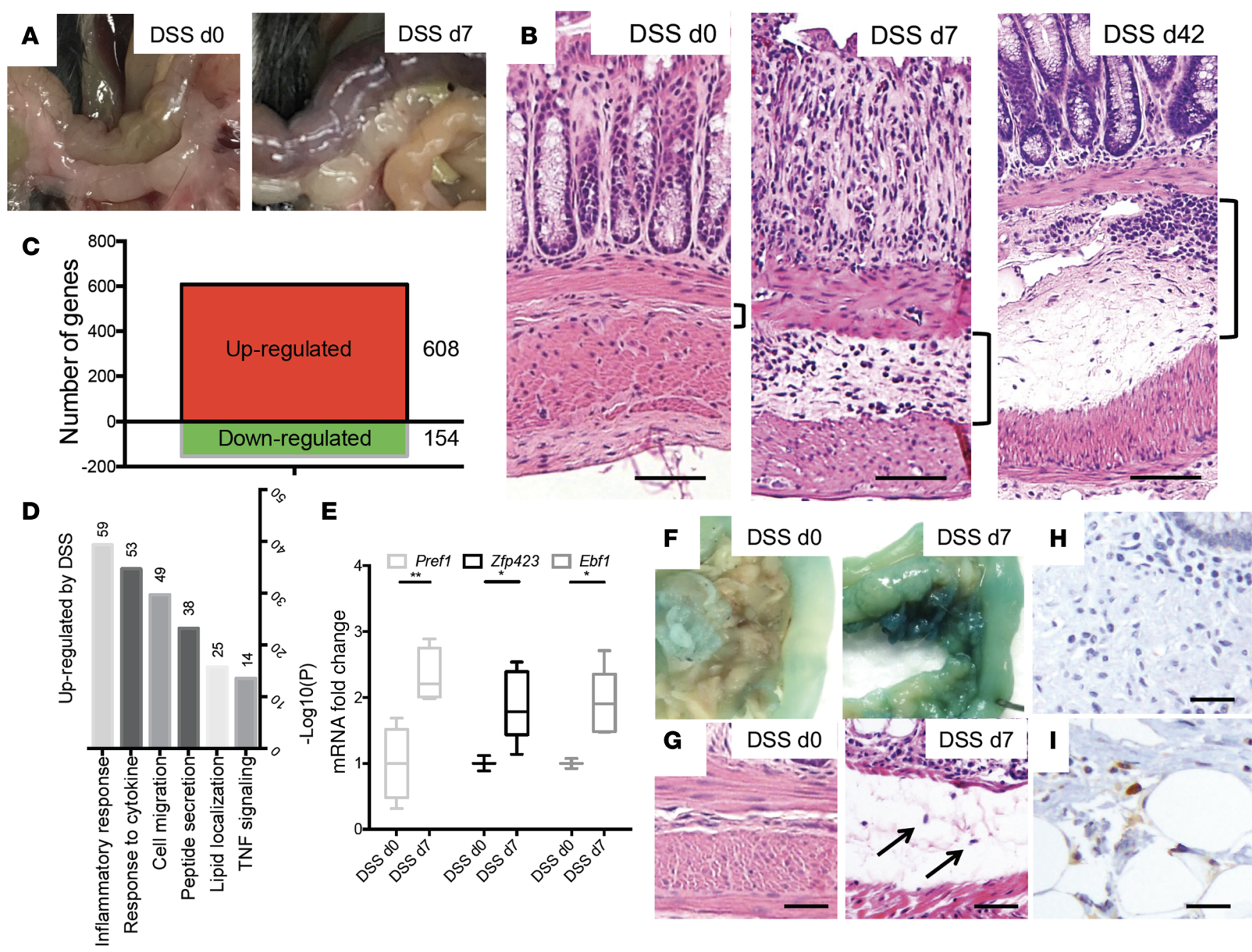

Figure 2. An increase in adipose occurs during experimental colitis. (A) Representative anatomical and (B) histological images of the distal colon from mice at day 0 and day 7 or 42 days after being provided with 3\% DSS in drinking water. Tissue was stained with hematoxylin and eosin. Brackets delineate submucosal region occupied by adipocytes. Scale bar: 50 microns. (C) Transcripts identified by RNA sequencing to be increased or decreased after 7 days of DSS (2-fold change vs. normal colon). (D) Gene ontology analysis of the upregulated genes by DSS treatment. (E) Reverse transcription qPCR (RTqPCR) of the relative abundance of transcripts for Pref1, Zfp423, and Ebf1, as normalized to $\beta$-actin ( $n=5$ mice/group). Data in $\mathbf{E}$ is represented using box-andwhisker plots, with boxes representing the IQR, lines representing the median value, and whiskers representing minimum and maximum values; ${ }^{*} P<$ 0.05 , ${ }^{* *} P<0.01$, and ${ }^{* * *} P<0.001$ (Student's $t$ test). (F and $\left.\mathbf{~}\right) \beta$-Gal staining of the Zfp423 $3^{\text {Lacz/+ }}$ mice colon 7 days after DSS. (H) Representative histology of uninvolved Crohn's disease human patient tissues stained with anti-PREF1/DLK antibody. Scale bar: 10 microns. (I) Representative histology of involved Crohn's disease human patient tissues stained with anti-PREF1/DLK antibody. Scale bar: 10 microns.

directly modulate several immune responses, we next examined the effect of hyaluronidase on the inflammatory response of both skin and colon.

In skin, both transgenic expression of Hyal1 and the administration of PEGPH20 resulted in a significant reduction in IL-6 despite active infection with $S$. aureus (Figure 5A). Consistent with the inhibition of reactive adipogenesis, the expression of the antimicrobial peptide Camp was also inhibited (Figure 5B). In the colon, an even more profound reduction in inflammation was observed after the addition of hyaluronidase. Mice with DSS-induced colitis had less inflammatory infiltrate when HA was digested by Hyall or PEGPH20 (Figure 5C). Hyaluronidase activity protected against the decrease in body weight seen at day 7 after DSS (Figure 5D).

RNA sequencing identified 453 genes whose expression was altered by hyaluronidase (Supplemental Tables 1 and 2), and the majority of these genes (420/453) that were inhibited were clustered in inflammatory, cytokine, immune response-related, and adipogenic pathways (Figure 5, E and F).

Hyaluronidase treatment also resulted in less infiltration of LY6G-positive neutrophils in the lamina 

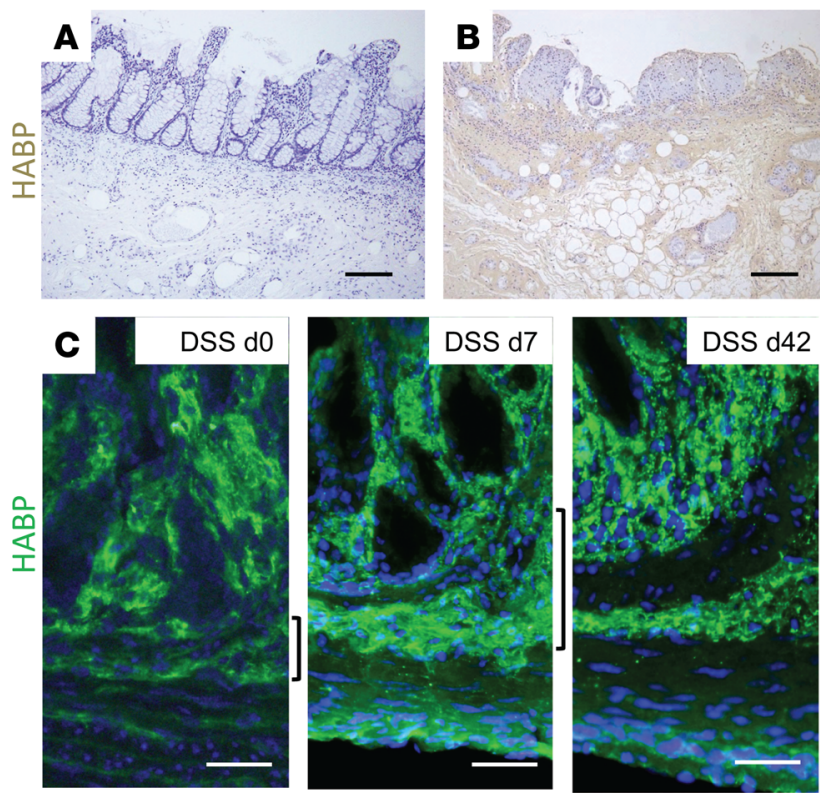

Figure 3. Hyaluronan accumulates during inflammation of the colon in humans and mice. (A) Representative histology of uninvolved tissue from Crohn's disease human patient tissues stained with hyaluronic acid binding proteins (HABP). Scale bar: 10 microns. (B) Representative histology of involved tissue from Crohn's disease human patient tissues stained with HABP. Scale bar: 10 microns. (C) Representative histological images of the distal colon from mice at day 0 and day 7 or 42 days after being provided with $3 \%$ DSS in drinking water to induce colitis. Tissue was stained with HABP. Brackets delineate submucosal region occupied by adipocytes. Scale bar: 50 microns.

propria of the colon after DSS (Figure 6, A and B) and led to lesser accumulation of macrophages and dendritic cells (Supplemental Figure 5, A-D). The effects of hyaluronidase on inflammation of the colon were similar to or greater than the effects of inhibition of adipogenesis by BADGE. TNF- $\alpha$ (Tnf $\alpha$ ) expression was suppressed by both hyaluronidase and BADGE, and a better histological disease score was observed in all groups (Figure 6, C and D). BADGE also decreased expansion of the submucosal adipose layer in the colon after DSS (Supplemental Figure 6A). However, in contrast to the decrease in inflammation, hyaluronidase activity increased endotoxins and bacteria detected in sera (Figure 6, E-G), an observation consistent with an increase in bacterial translocation across the injured colon epithelial lumen. These observations are consistent with hyaluronidase inhibiting the proinflammatory effects and antimicrobial defense functions of reactive adipogenesis.

\section{Discussion}

In this study, we hypothesized that the accumulation of HA and associated development of adipose tissue may have a physiological role in inflammation. This hypothesis was based on recent observations that adipogenesis occurs in the dermis in response to tissue damage $(16,21)$ at the same time that HA turnover occurs $(3,6,22-25)$. To test this hypothesis, we examined the relationship between HA and adipogenesis during injury of 2 major epithelial organs: the skin and gut. Digestion of HA by hyaluronidase suppressed reactive adipogenesis both in vitro and in vivo. Both skin and colon showed a decrease in inflammation and tissue damage upon addition of hyaluronidase. Moreover, despite an increase in bacterial invasion, hyaluronidase-treated animals improved in their evidence of disease as measured by more stable weight and lower histological disease score. These observations suggest that HA has a major influence on the response of preadipocytes to epithelial damage and suggests for the first time to our knowledge that reactive adipogenesis is important not only to skin defense but also to defense of the colon following epithelial injury.

IBD is characterized by chronic inflammation of the gastrointestinal tract and has been associated with poor quality of life and frequent complications requiring hospitalization and surgical procedures. In 2015, 3.1 million (1.3\%) US adults received a diagnosis of IBD (26). Current therapies for IBD typically target neutralization of inflammatory cytokines, blockade of receptors, or inhibition of inflammatory cell functions. Despite current approaches, it is still difficult to control disease severity and maintain quality of life. One important phenotype of IBD that may offer an opportunity for gaining increased understanding of the disease is that up to $40 \%$ of individuals with inflammatory diseases of the colon have extraintestinal manifestations. Foremost in these extraintestinal symptoms are skin or oral disorders, such as erythema nodosum, pyoderma gangrenosum, and aphthous stomatitis (27-29). The presence of diseases associated with IBD at sites far from the gut support alternative hypotheses that IBD is a systemic disorder of circulating bone marrow-derived immunocytes, a consequence of dysbiosis of the microbiome (30), or a generalized 


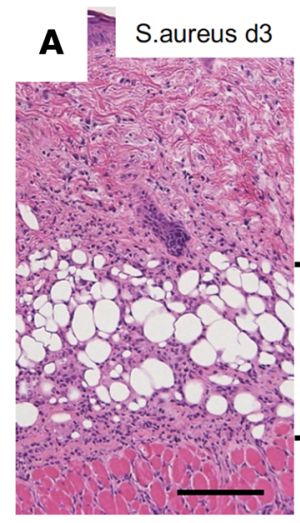

C
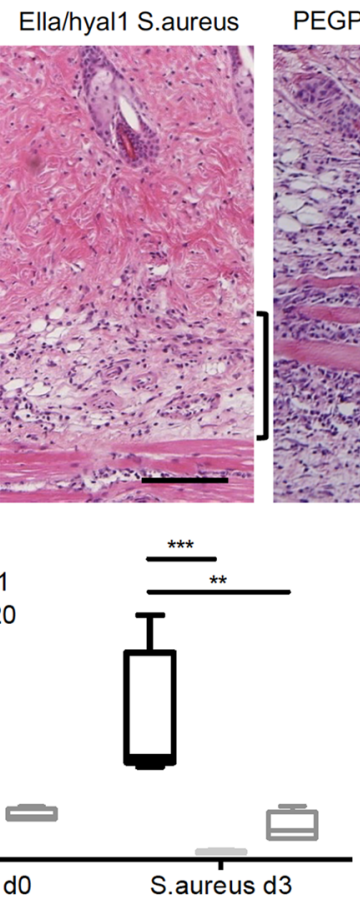
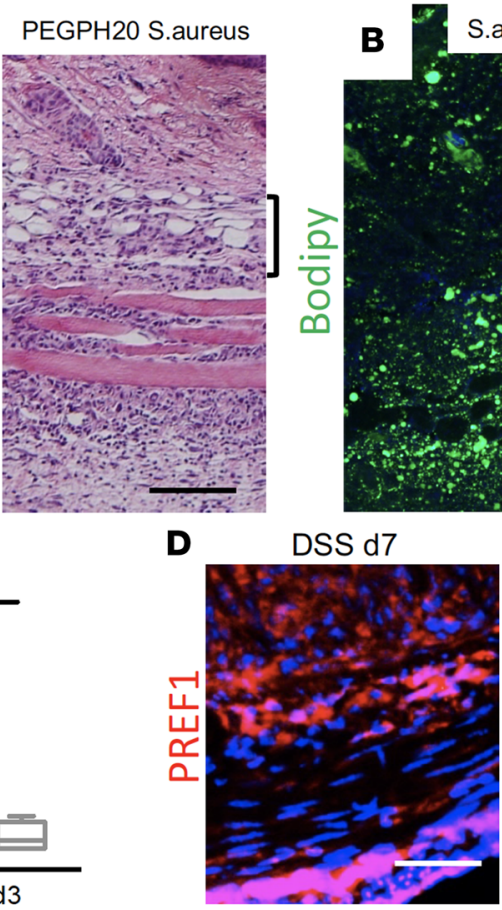

$\mathbf{F}$

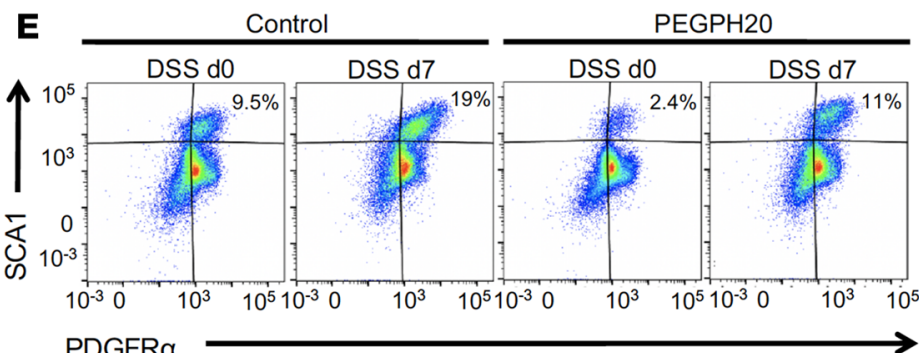

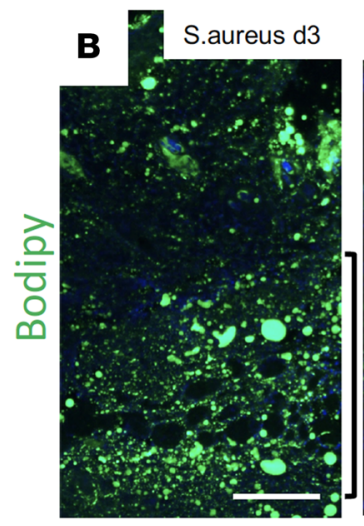
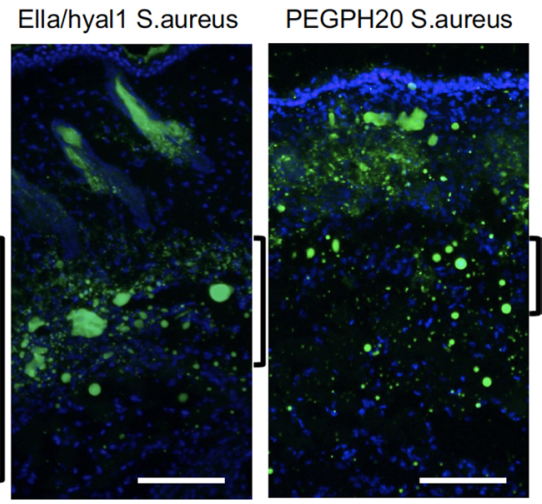

Ella/Hyal1 DSS d7
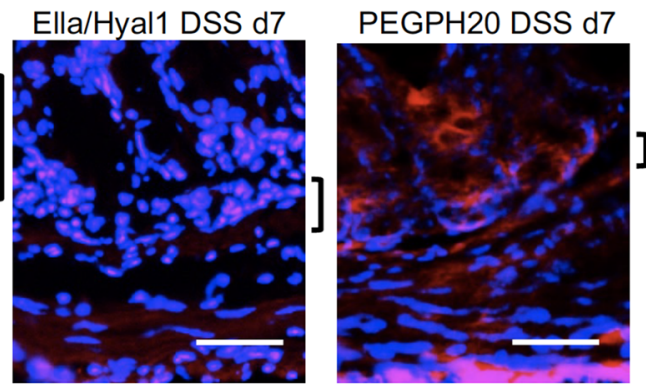

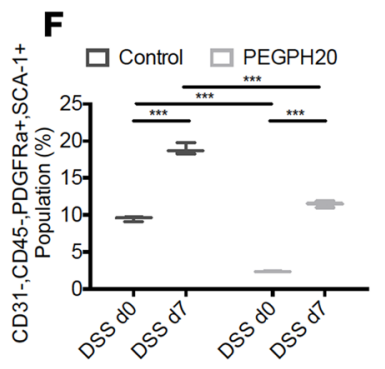

G

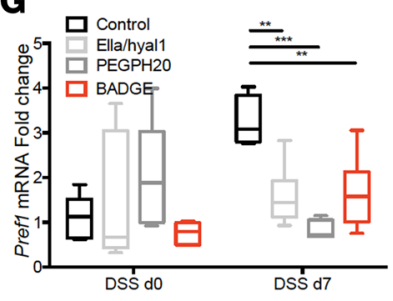

Figure 4. Reactive adipogenesis is inhibited by hyaluronidase. (A) Representative H\&E histology of skin 3 days after infection with S. aureus. WT mice are compared with non-WT mice following the addition of hyaluronidase by transgenic expression during early embryogenesis (Ella/hyal1) or administration of PEGPH2O. (B) Lipid droplet staining of frozen sections similar to A. (C) mRNA abundance from skin measured by qPCR of $C e b p \alpha(n=6$ mice/ group). (D) Representative sections of distal colon from control, Ella/hyal1-injected, and PEGPH20-injected mice 7 days after being provided with $3 \%$ DSS colitis in drinking water. Tissue was stained with anti-PREF1/DLK antibodies. Brackets delineate submucosal region occupied by adipocytes. (E and F) Flow cytometry analysis of single-cell suspensions from the colon lamina propria showing expression of PDCFR- $\alpha$ and SCA1 from control, PECPH20, DSS d7, and PECPH20-treated DSS d7 mice. Cells were gated on CD31-negative, CD45-negative. Numbers represent the percentage of the cells in the indicated gate. (C) mRNA abundance measured by qPCR of Pref1 ( $n=4$ control or BADGE and PEGPH20 or 6 Ella/Hyal1 mice/group). Scale bar: 50 microns. Data in $\mathbf{C}$ and $\mathbf{G}$ are represented using box-and-whisker plots, with boxes representing the IQR, lines representing the median value, and whiskers representing minimum and maximum values, whereas data in $\mathbf{F}$ is represented as mean $\pm \mathrm{SEM}$; ${ }^{* *} P<0.01$ and ${ }^{* * *} P<0.001$ (Student's $t$ test). d0, day 0 ; d3, day 3 ; d7, day 7 .

disorder of epithelial function. Indeed, the close interplay of microbes with multiple tissue and cell types supports the hologenome $(31,32)$ theory of evolution that suggests the function of both human and microbial cell types is necessary for tissue homeostasis. Appropriate function of the epithelial barrier is necessary to regulate the interactions between microbes and the host $(33,34)$ and maintain health.

The biological significance of increased fat accumulation surrounding sites of epithelial injury to the colon was previously unknown. Though visceral adipose tissue has long been speculated to be a component of the innate immune system of the gut (35), and several descriptions of the production of "adipokines" from fat such as leptin, adiponectin, and cytokines have been reported (36), the contribution of adipokines to IBD has been controversial $(20,37)$. We propose here that recent observations of reactive adipogenesis in the skin can be applied to also understand the significance of adipogenesis in the intestine. In skin, DWAT expands during wound repair and infection $(13,14,16,38,39)$. The capacity of these activated preadipocytes to produce antimicrobial peptides is critical to skin defense in mice $(16,40,41)$. This study hypothesized that an adipogenic response could also be observed in a mouse model of colitis. Our findings showed that DSS induced histological evidence of increased adipose, increased expression 
A

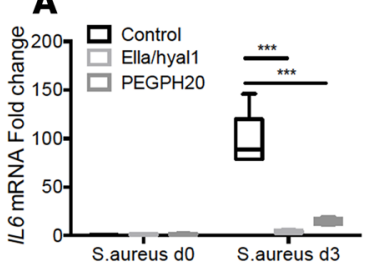

B

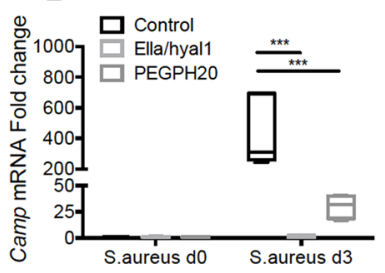

D

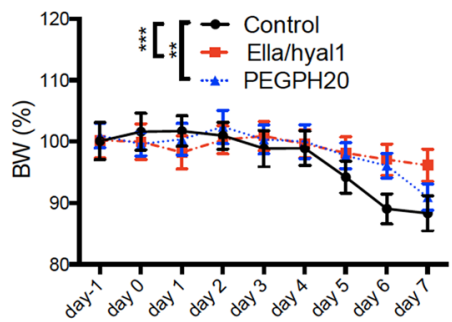

C

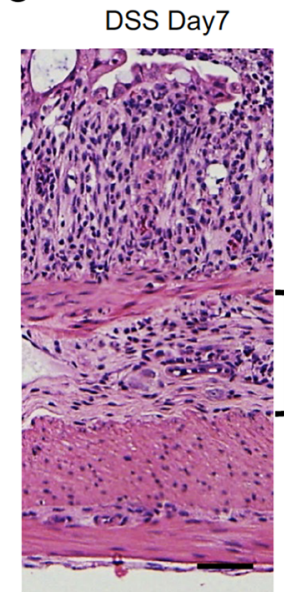

E
Ella/Hyal1 DSS d7

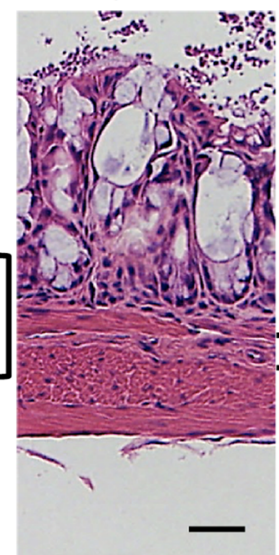

Down-regulated by PEGPH2O

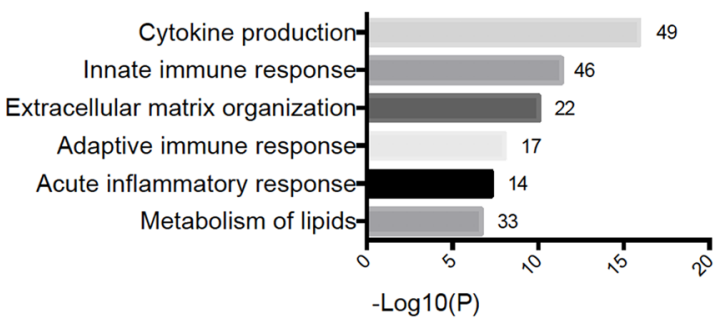

$\mathbf{F}$
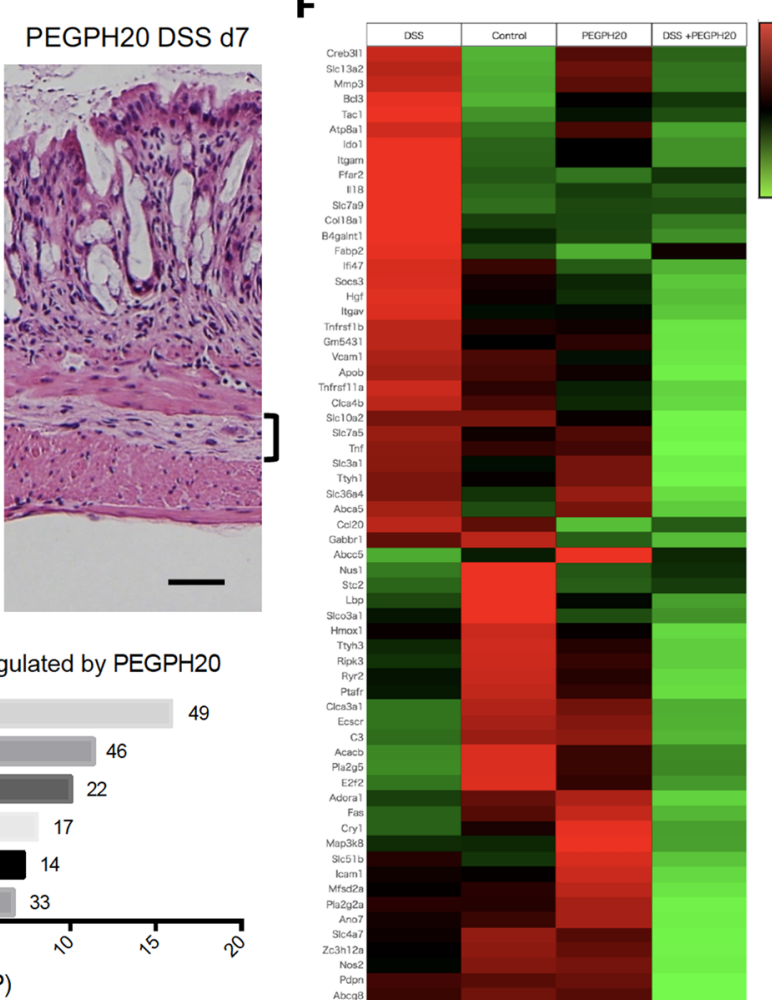

Figure 5. Hyaluronidase inhibits inflammation of the skin and colon. (A and B) mRNA abundance of skin 3 days after infection with S. aureus measured by qPCR of IL-6 and Camp ( $n=6$ mice/group). WT mice are compared with Ella/hyal1 mice or mice after IV administration of PEGPH20. (C) Representative H\&E histology at day 7 after DSS in distal colon sections of WT, Ella/hyal1, and PEGPH2O-injected mice. (D) Daily body weight measurements normalized to original body weight in mice during administration of DSS ( $n=5$ mice/group). Statistics are performed by 1-way ANOVA multiple comparison test. Data in $\mathbf{A}$ and $\mathbf{B}$ are represented using box-and-whisker plots, with boxes representing the IQR, lines representing the median value, and whiskers representing minimum and maximum values, whereas data in $\mathbf{D}$ is represented as mean $\pm \mathrm{SEM}$; ${ }^{* *} P<0.01$ and ${ }^{* *} P<0.001$ (Student's $t$ test). (E and $\mathbf{F}$ ) RNA sequencing was performed on tissue extracted from the submucosal regions of the colon of control mice, mice treated with PEGPH20, mice treated for 7 days with DSS, or mice treated with DSS and PEGPH2O. (E) Gene ontology analysis of transcripts induced by DSS that were inhibited by greater than 2-fold with PEGPH2O. (F) Hierarchical clustering heat map of innate immune response-related genes.

of genes associated with adipogenesis, and increased accumulation of HA. These observations in mice were consistent with the "creeping fat" phenomenon in human colitis and suggested that the murine DSS colitis model is an appropriate method to study this phenomenon.

We explored the potential for hyaluronidase to inhibit adipogenesis because of the targeted nature of this enzyme, its reversible effects, and the known association of HA as a reaction to injury $(3,42,43)$. Both skin and intestine have abundant HA in the epithelial and subepithelial layers $(2,44)$, and this HA rapidly turns over, with as much as a third of HA replaced daily. HA accumulation was observed in inflamed submucosal layers from samples obtained from human IBD and similarly occurred in cultured preadipocytes when triggered to undergo differentiation to adipocytes. Addition of a stable, soluble form of hyaluronidase (PEGPH20) successfully digested HA and suppressed adipogenesis in cultured preadipocytes and effectively digested HA in the colon and skin. To validate subsequent interpretations of the consequences of this event, we applied an independent technique for digestion of HMW-HA by transgenic systemic expression of Hyal1. The success of both independent approaches with different enzymes supported the conclusion that the hyaluronidase activity itself, rather than an alternative function of $\mathrm{PH} 20$ or Hyall, was responsible for the observed phenotype.

No effect on the health or behavior of mice was seen at baseline following the administration of hyaluronidase to mice in this study or in prior studies (7). Interestingly, despite the lack of an obvious phenotype at baseline, FACS analysis found that PEGPH20 injection decreased the proportion of preadipocytes, DCs, and macrophages in the colonic submucosa. Furthermore, both approaches to the administration of hyaluronidase resulted in a significant decrease in the inflammatory response to injury. Hyaluronidase decreased expression of IL- 6 and TNF- $\alpha$ and decreased the inflammatory cell infiltrate. Inhibiting adipogenesis by 
A

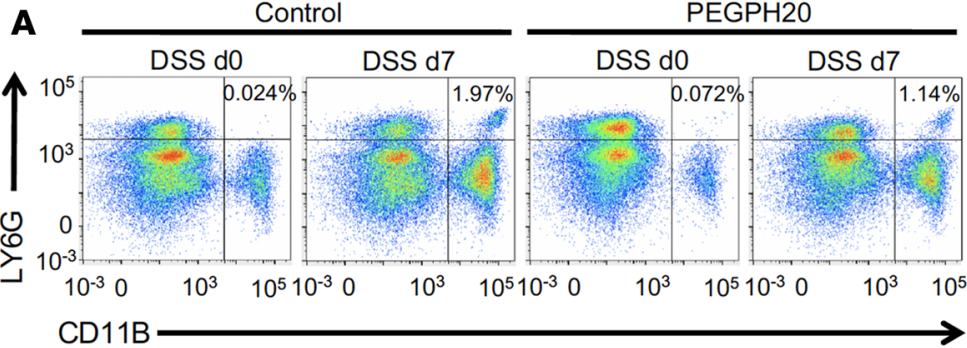

B

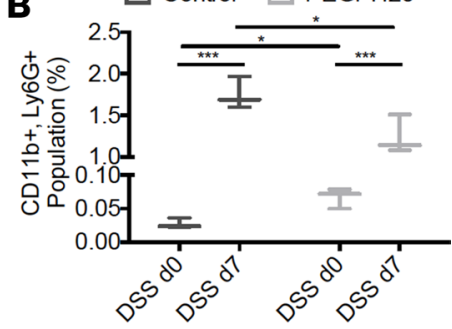

C

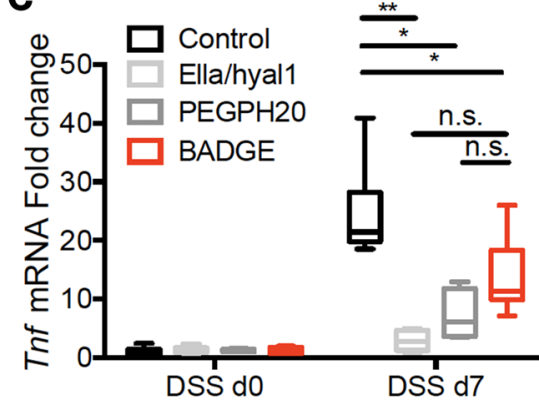

D

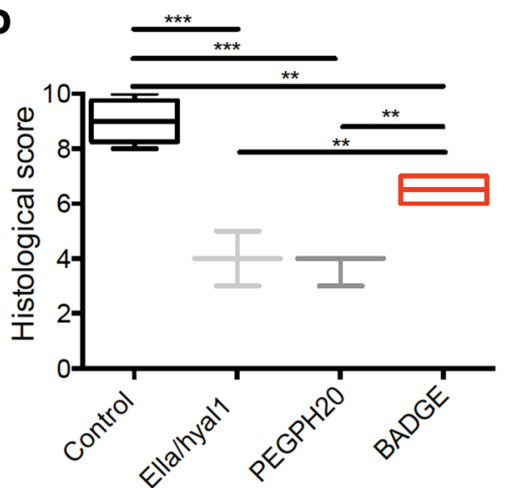

E

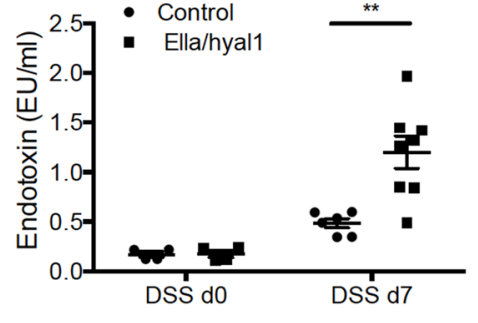

F

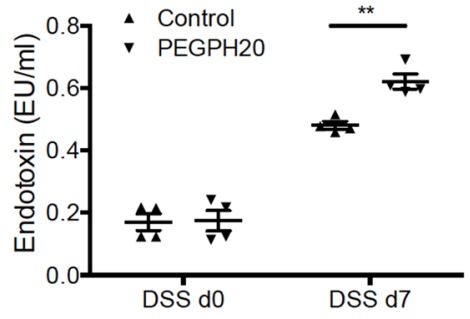

G

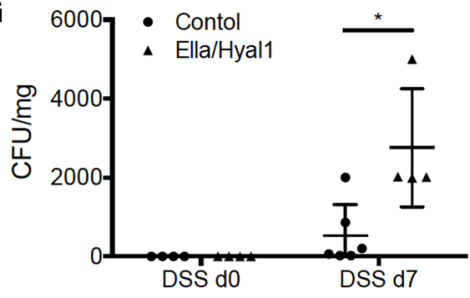

Figure 6. Hyaluronidase inhibits TNF- $\alpha$ expression, increases bacterial translocation, and improves disease score in colitis. (A and B) Flow cytometry analysis of single-cell suspensions from the colon lamina propria showing expression of LY6C and CD11B from control mice at DSS d0 and DSS d7 and mice treated with PECPH2O at DSS dO and DSS d7. Numbers represent the percentage of the cells in the indicated gate. (C) RTqPCR measurements of the relative abundance of TNF- $\alpha$. mRNA expression was normalized to $\beta$-actin ( $n=5$ mice/group). (D) The histological scores of day 7 samples were measured by the Crohn's Disease Activity Index. (E and F) Endotoxin concentration in the serum from WT and Ella/hyal1 mice at day 0 or treated with DSS for 7 days ( $n=6$ control, $n=8$ Ella/ hyal1, and $n=4$ PEGPH20 mice/group). (C) Systemic bacteremia detected after colitis in inflamed tissue from WT and Ella/hyal1 mice ( $n=4$ mice/group). Representative histology of distal colon sections in colitis of WT, Ella/hyal1, PEGPH2O-injected, and BADCE-injected mice were stained with H\&E. Data in C and D are represented using box-and-whisker plots, with boxes representing the IQR, lines representing the median value, and whiskers representing minimum and maximum values, whereas data in $\mathbf{B}$ and $\mathbf{E}-\mathbf{G}$ are represented as mean $\pm \mathrm{SEM} ;{ }^{*} P<0.05,{ }^{* *} P<0.01$, and ${ }^{* * *} P<0.001$ (Student's $t$ test). EU, endotoxin units.

administration of BADGE also decreased cytokine expression and supported the contribution of adipogenesis to inflammation of the colon, but the magnitude of this inhibition was less than that seen following administration of hyaluronidase. Furthermore, despite an apparent decrease in tissue damage of the colon after hyaluronidase administration, an increase in serum endotoxin and bacterial counts was observed. This finding is consistent with the inhibition of antimicrobial peptide (Camp) expression associated with decreased adipogenesis. However, no significant effect of BADGE was seen on bacterial translocation or endotoxin levels (Supplemental Figure 6, B and C). It is therefore possible that the effects of hyaluronidase on tissue inflammation may involve additional mechanisms that are independent of the action to inhibit adipogenesis. This may involve changes in sentinel dendritic cells similar to the effects of hyaluronidase on dermal dendritic cells in the skin, as we have published previously (7).

Taken together, these observations show for the first time to our knowledge that digestion of HA has the potential to inhibit reactive adipogenesis after injury to the colon and skin and protect against 
inflammatory tissue damage. These findings advance understanding of the role of HA and adipocytes in host defense. These findings also further illustrate commonalities in inflammatory events at the skin and colon and explain that the physiological significance of increased adipose tissue in IBD is the innate immune defense process of reactive adipogenesis.

\section{Methods}

Chemicals and reagents. PEGPH20 was provided by Halozyme Therapeutics Inc. DSS was purchased from MP Biomedicals Inc. Rabbit anti-CAMP antibodies were made from our lab as described previously (45); rabbit anti-PREF1/DLK antibodies are from Abcam; BODIPY fluorophore dye was purchased from Thermo Fisher Scientific. HA binding protein was purchased from MilliporeSigma. Human-Hyal1, mouse-Hyal1, Hyal2, KIAA1199, TMEM2, HAS1, HAS2, HAS3, EBF1, ZFP521, ZFP423, Pref1, PPAR $\gamma$, Adipoq, CEBP- $\alpha$, CAMP, IL-6, TNF- $\alpha$, and TaqMan Gene Expression Assay were purchased from Applied Biosystems.

Animals and animal care. Transgenic mice for conditional overexpression of human hyaluronidase 1 (in C57BL/6 background) were generated in our laboratory, as described previously, by combining a constitutive promoter and a loxP-flanked GFP reporter upstream of hyaluronidase 1 (CAG-loxP-GFPstop-loxP-Hyal1). Cross-breeding with E2a-Cre mice (Ella/HYAL1 mice) for early embryonic expression of Cre enabled the promoter to drive the expression of the downstream hyaluronidase gene. WT mice (C57BL/6 mice) were obtained from The Jackson Laboratory. All animal experiments were approved by the University of California, San Diego, Institutional Animal Care and Use committee. For all animal studies, animals were randomly selected without formal prerandomization, and quantitative measurements were done without the opportunity for bias.

Bacterial strains. S. aureus strain USA300 (46) is a predominant community-associated MRSA strain and was provided by Victor Nizet (Skaggs School of Pharmacy and Pharmaceutical Sciences, UCSD, La Jolla, California, USA).

Mouse model of S. aureus skin infection. Skin infection experiments were done as described before (47). S. aureus strain USA300/MRSA was used for infection. In brief, the backs of sex-matched and age-matched (8-week to 12-week) C57BL/6 WT or C57BL/6 Ella/Hyal1 mice were shaved and hair removed by chemical depilation (Nair), then injected subcutaneously with $100 \mu \mathrm{l}$ of a midlogarithmic growth phase of $S$. aureus $\left(2 \times 10^{6} \mathrm{CFU}\right.$ of bacteria) in PBS. Mice were sacrificed after day 3, and an 8-mm skin punch biopsy comprising the center of the injection site was harvested. Infected skin surrounding the infection center (6-8 $\mathrm{mm})$ void of center abscess was carefully dissected out for RNA extraction or CFU determination. Skin biopsies were homogenized in $1 \mathrm{ml}$ TRIzol (Life Technologies Corporation) (for RNA) or PBS (for CFU counting) with 2-mm zirconia beads in a Mini-Beadbeater-16 (BioSpec Products). To count CFU, homogenized skin samples were serially diluted, plated onto Tryptic Soy Agar (Sigma-Aldrich), and enumerated after 18 hours to quantify the CFU per gram of tissue. For some experiments, PEGPH20 (1 mg/kg) was injected intravenously starting from 1 day before infection once, and the vehicle $(10 \mu \mathrm{mol} / \mathrm{L}$ histidine, $130 \mu \mathrm{mol} / \mathrm{L} \mathrm{NaCl}$, at $\mathrm{pH} 6.5)$ was used as a control.

Mouse model of DSS colitis. Colitis experiments were done as described before (48). WT or Ella/hyal1 mice were provided 3\% DSS in their drinking water for 7 days, and body weight was measured every day. For long-term exposure, mice were provided 3\% DSS in drinking water for 7 days followed by a 7-day period of normal water without DSS. This cycle of 7 days on DSS and 7 days off was repeated 3 times. For HA digestion by PEGPH20, mice were intravenously injected with PEGPH20 (1 mg/kg) every 3 days 24 hours before the start of DSS water feeding. For adipogenesis inhibition by PPAR $\gamma$ inhibitor, mice were intraperitoneally injected $120 \mathrm{mg} / \mathrm{kg}$ every day 48 hours before the start of DSS water feeding. Mice were sacrificed and distal colon, mesenteric fat, and blood were collected for RNA, histology, bacterial culture assay, and toxin analysis. Histological scores were measured as previously described (49-51).

Preadipocyte culture. The preadipocyte cell line 3T3-L1 was purchased from ATCC (CL-173) lot 59239597. 3T3-L1 cells were grown in preadipocyte proliferation medium (Cell Applications Inc.), and P4 P7 cells were used for in vitro differentiation. To induce differentiation, 2 days post-confluent 3T3 L1 cells were switched to an adipocyte differentiation medium containing insulin, dexamethasone, and IBMX (Cell Applications Inc.), and the medium was changed to fresh medium at day 2 and 4 . To generate differentiated adipocytes for comparison studies between 3T3-L1 preadipocytes and adipocytes, 3T3-L1 cells were differentiated for 3 days followed by 2 days of culture in preadipocyte growth medium (Cell Applications Inc.) before RNA or protein extraction. To analyze cell proliferation, cells were fixed, and sulforhodamine B assay was performed (52). 
RTqPCR analyses. RTqPCR was used to determine the mRNA abundance as described previously (53). Total cellular RNA was extracted using the PureLink RNA Mini Kit (Life Technologies Corporation), and mRNA was purified by using Dynabeads mRNA Purification Kit (Life Technologies Corporation). Using an iScript cDNA synthesis kit (Bio-Rad Laboratories Inc.), 100 ng of mRNA was reverse transcribed to cDNA. Quantitative, real-time PCR was performed on the CFX96 real-time system (Bio-Rad Laboratories Inc.) using a predeveloped TaqMan Gene Expression Assay (Applied Biosystems). The expression of $\beta$-actin gene was used as a housekeeping gene to normalize data.

RNA sequencing. Purified RNA from colon lamina propria was submitted to the UCSD Institute for Genomic Medicine core facility for library preparation and high-throughput, next-generation sequencing. Libraries were constructed using TruSeq Stranded mRNA Library Prep Kits (Illumina) and run on a HiSeq 2500 instrument (Illumina). Raw data were analyzed using Partek Flow and Partek Genomics Suite software to determine transcript abundance and differentially expressed genes between samples. Gene ontology analysis was performed using Metascape (http://metascape.org, ref. 54).

Histology and immunohistochemistry. Tissue biopsies were directly embedded in OCT compound or paraffin. Paraffin-embedded tissues were used for H\&E staining, and frozen sections were fixed in 4\% paraformaldehyde for 20 minutes or 100\% acetone before immunofluorescence staining. For immunohistochemistry, fixed and permeabilized frozen tissue sections were blocked with Image-iT FX reagent (Invitrogen) before incubating with primary antibodies followed by appropriate 488- or 568-coupled secondary antibodies. Nuclei were counterstained with DAPI. All images were taken with an Olympus BX41 microscope (widefield) or Zeiss LSM510 confocal microscope as appropriate.

Flow cytometry analyses. Colon collected from control or DSS-treated mice was cut into small pieces, then digested with $2.5 \mathrm{mg} / \mathrm{ml}$ collagenase $\mathrm{D}$ and $30 \mathrm{ng} / \mathrm{mL}$ DNAse 1 for 2 hours at $37^{\circ} \mathrm{C}$, then filtered through a $30-\mu \mathrm{m}$ filter to generate single-cell suspension for FACS analyses. Cells were then stained with Zombie Violet viability dye (BioLegend, 423114), blocked with antimouse CD16/CD32 (eBioscience, 14016185), and stained with antibody cocktails for preadipocytes or immune cells. The antibody cocktail for preadipocytes includes AF488-SMA (eBioscience, 53976082), PECy7-CD45 (BioLegend, 147704), PerCy5.5-CD31 (BioLegend, 102522), PE-Thy1 (BioLegend, 105308), APC-PDGFR- $\alpha$ (eBioscience, 17140181), BV605SCA1 (BioLegend, 108133), and AF700-CD24 (BioLegend, 108136). The antibody cocktail for immune cells includes PECy7-CD11b (BioLegend, 101216), FITC-Ly6G (eBioscience, 11593182), PE-F4/80 (eBioscience, 12480182), APC-CD11C (BioLegend, 117310), AF700-MHCII (eBioscience, 56532182) and APCCy7-CD3 (BioLegend, 100222). FACS analyses for surface expression of preadipocyte or immune cell markers were performed by the Becton, Dickinson and Company FACSCanto RUO machine and analyzed by FlowJo V10 software. Dead cells stained positive with Zombie Violet dye were excluded from the analyses.

Serum endotoxin analyses. Mouse serum was separated from mouse whole blood using serum separator tubes (Becton, Dickinson and Company). Mouse serum endotoxin levels were quantified by ToxinSensor Chromogenic LAL Endotoxin Assay Kit (GenScript).

$H A$ analysis. Glycosaminoglycans, including HA, were extracted from 3T3-L1 supernatant and murine skin and colon as previously described (7). Samples were homogenized and treated overnight with protease $(0.16$ $\mathrm{mg} / \mathrm{ml}$; Sigma-Aldrich) to degrade protein, followed by purification by anion exchange chromatography using DEAE Sephacel (Amersham Biosciences). Columns were washed with a low-salt buffer $(0.15 \mathrm{M} \mathrm{NaCl}$ in 20 $\mu \mathrm{M}$ sodium acetate; $\mathrm{pH}$ 6.0) and eluted with $1 \mathrm{M} \mathrm{NaCl}$. Glycans were desalted by PD-10 (GE Healthcare). HA concentrations were measured with ELISA DuoSet (R\&D Systems). The size distribution of HA was analyzed by agarose gel electrophoresis (55). The HA sample was mixed with Tris base, acetic acid, and EDTA buffer containing $2 \mathrm{M}$ sucrose and electrophoresed at $2 \mathrm{~V} / \mathrm{cm}$ for 10 hours at room temperature. The gel was stained overnight under a light-protective cover at room temperature in a solution containing $0.005 \%$ Stains-All (Sigma-Aldrich) in 50\% ethanol and destained in water. Hyalose ladders (Hyalose, LLC) were used for standards.

Accession numbers. RNA sequencing data have been deposited in the Gene Expression Omnibus (GEO GSE119826).

Statistics. Experiments were repeated at least 3 times with similar results. Statistical significance was determined using Student's unpaired 2-tailed $t$ test, or 1-way ANOVA multiple comparison test, as indicated in the figure legends $\left({ }^{*} P<0.05,{ }^{* *} P<0.01\right.$, and $\left.{ }^{* * *} P<0.001\right)$.

Study approval. All animal experiments were approved by the University of California, San Diego, Institutional Animal Care and Use committee. For all animal studies, animals were randomly selected without formal prerandomization, and quantitative measurements were done without the opportunity for bias. 


\section{Author contributions}

TD and RLG designed and performed experiments, analyzed data, and wrote the manuscript. LZ designed and performed experiments and analyzed data. TN, CAA, JAS, RDP, HT, and MF designed experiments and analyzed data.

\section{Acknowledgments}

TD and RLG were funded by NIH P01HL107150. LZ, TN, and RLG were funded by NIH R01AR069653 and R01AI052453. PEGPH20 was a gift from Halozyme Therapeutics Inc.

Address correspondence to: Richard L. Gallo, Department of Dermatology, MC0869, University of California, San Diego, 9500 Gilman Dr, La Jolla, California, USA, 92093-0869. Phone: 858.822.4608; Email: rgallo@ucsd.edu.

1. Laurent TC, Laurent UB, Fraser JR. The structure and function of hyaluronan: An overview. Immunol Cell Biol. 1996;74(2):A1-A7.

2. Wang C, Tammi M, Guo H, Tammi R. Hyaluronan distribution in the normal epithelium of esophagus, stomach, and colon and their cancers. Am J Pathol. 1996;148(6):1861-1869.

3. Jiang D, Liang J, Noble PW. Hyaluronan as an immune regulator in human diseases. Physiol Rev. 2011;91(1):221-264.

4. Wang A, de la Motte C, Lauer M, Hascall V. Hyaluronan matrices in pathobiological processes. FEBS J. 2011;278(9):1412-1418.

5. Cyphert JM, Trempus CS, Garantziotis S. Size matters: molecular weight specificity of hyaluronan effects in cell biology. Int $J$ Cell Biol. 2015;2015:563818.

6. Jiang D, et al. Regulation of lung injury and repair by Toll-like receptors and hyaluronan. Nat Med. 2005;11(11):1173-1179.

7. Muto J, et al. Hyaluronan digestion controls DC migration from the skin. J Clin Invest. 2014;124(3):1309-1319.

8. Hill DR, Kessler SP, Rho HK, Cowman MK, de la Motte CA. Specific-sized hyaluronan fragments promote expression of human $\beta$-defensin 2 in intestinal epithelium. J Biol Chem. 2012;287(36):30610-30624.

9. Kim Y, Kessler SP, Obery DR, Homer CR, McDonald C, de la Motte CA. Hyaluronan 35kDa treatment protects mice from Citrobacter rodentium infection and induces epithelial tight junction protein ZO-1 in vivo. Matrix Biol. 2017;62:28-39.

10. de la Motte CA, Kessler SP. The role of hyaluronan in innate defense responses of the intestine. Int J Cell Biol. 2015;2015:481301.

11. Liang J, et al. Hyaluronan and TLR4 promote surfactant-protein-C-positive alveolar progenitor cell renewal and prevent severe pulmonary fibrosis in mice. Nat Med. 2016;22(11):1285-1293.

12. Ji E, et al. Inhibition of adipogenesis in 3T3-L1 cells and suppression of abdominal fat accumulation in high-fat diet-feeding C57BL/6J mice after downregulation of hyaluronic acid. Int J Obes (Lond). 2014;38(8):1035-1043.

13. Horsley V, Watt F. Repeal and replace: adipocyte regeneration in wound repair. Cell Stem Cell. 2017;20(4):424-426.

14. Plikus MV, et al. Regeneration of fat cells from myofibroblasts during wound healing. Science. 2017;355(6326):748-752.

15. Guerrero-Juarez CF, Plikus MV. Emerging nonmetabolic functions of skin fat. Nat Rev Endocrinol. 2018;14(3):163-173.

16. Zhang LJ, et al. Innate immunity. Dermal adipocytes protect against invasive Staphylococcus aureus skin infection. Science. 2015;347(6217):67-71.

17. Driskell RR, et al. Distinct fibroblast lineages determine dermal architecture in skin development and repair. Nature. 2013;504(7479):277-281.

18. Hudak CS, Sul HS. Pref-1, a gatekeeper of adipogenesis. Front Endocrinol (Lausanne). 2013;4:79.

19. Gao H, et al. Early B cell factor 1 regulates adipocyte morphology and lipolysis in white adipose tissue. Cell Metab. 2014;19(6):981-992.

20. Karrasch T, Schaeffler A. Adipokines and the role of visceral adipose tissue in inflammatory bowel disease. Ann Gastroenterol. 2016;29(4):424-438.

21. Gallo RL, Hooper LV. Epithelial antimicrobial defence of the skin and intestine. Nat Rev Immunol. 2012;12(7):503-516.

22. West DC, Hampson IN, Arnold F, Kumar S. Angiogenesis induced by degradation products of hyaluronic acid. Science. 1985;228(4705):1324-1326.

23. Mohamadzadeh M, DeGrendele H, Arizpe H, Estess P, Siegelman M. Proinflammatory stimuli regulate endothelial hyaluronan expression and CD44/HA-dependent primary adhesion. J Clin Invest. 1998;101(1):97-108.

24. Meran S, et al. Involvement of hyaluronan in regulation of fibroblast phenotype. J Biol Chem. 2007;282(35):25687-25697.

25. Mack JA, et al. Enhanced inflammation and accelerated wound closure following tetraphorbol ester application or full-thickness wounding in mice lacking hyaluronan synthases Has1 and Has3. J Invest Dermatol. 2012;132(1):198-207.

26. Dahlhamer JM, Zammitti EP, Ward BW, Wheaton AG, Croft JB. Prevalence of inflammatory bowel disease among adults aged $\geq 18$ years - United States, 2015. MMWR Morb Mortal Wkly Rep. 2016;65(42):1166-1169.

27. van Wijk F, Cheroutre H. Mucosal T cells in gut homeostasis and inflammation. Expert Rev Clin Immunol. 2010;6(4):559-566.

28. Vavricka SR, et al. Frequency and risk factors for extraintestinal manifestations in the Swiss inflammatory bowel disease cohort. Am J Gastroenterol. 2011;106(1):110-119.

29. Huang BL, Chandra S, Shih DQ. Skin manifestations of inflammatory bowel disease. Front Physiol. 2012;3:13.

30. Allegretti J, Eysenbach LM, El-Nachef N, Fischer M, Kelly C, Kassam Z. The current landscape and lessons from fecal microbiota transplantation for inflammatory bowel disease: past, present, and future. Inflamm Bowel Dis. 2017;23(10):1710-1717.

31. Reshef L, Koren O, Loya Y, Zilber-Rosenberg I, Rosenberg E. The coral probiotic hypothesis. Environ Microbiol. 2006;8(12):2068-2073.

32. Carrier TJ, Reitzel AM. The hologenome across environments and the implications of a host-associated microbial repertoire. 
Front Microbiol. 2017;8:802.

33. Gallo RL. Human skin is the largest epithelial surface for interaction with microbes. J Invest Dermatol. 2017;137(6):1213-1214.

34. Zhang M, Sun K, Wu Y, Yang Y, Tso P, Wu Z. Interactions between intestinal microbiota and host immune response in inflammatory bowel disease. Front Immunol. 2017;8:942.

35. Karrasch T, Schmid A, Kopp A, Obermeier F, Hofmann C, Schäffler A. Impact of toll-like-receptor-9 (TLR9) deficiency on visceral adipose tissue adipokine expression during chronic DSS-induced colitis in mice. Exp Clin Endocrinol Diabetes. 2015;123(2):80-87.

36. Kopp A, et al. Innate immunity and adipocyte function: ligand-specific activation of multiple Toll-like receptors modulates cytokine, adipokine, and chemokine secretion in adipocytes. Obesity (Silver Spring). 2009;17(4):648-656.

37. Dworzanski T, et al. Influence of the peroxisome proliferator-activated receptor gamma (PPAR- $\gamma$ ) agonist, rosiglitazone and antagonist, biphenol-A-diglicydyl ether (BADGE) on the course of inflammation in the experimental model of colitis in rats J Physiol Pharmacol. 2010;61(6):683-693.

38. Wernstedt Asterholm I, et al. Adipocyte inflammation is essential for healthy adipose tissue expansion and remodeling. Cell Metab. 2014;20(1):103-118.

39. Alexander CM, et al. Dermal white adipose tissue: a new component of the thermogenic response. J Lipid Res. 2015;56(11):2061-2069.

40. Zhang LJ, Gallo RL. Antimicrobial peptides. Curr Biol. 2016;26(1):R14-R19.

41. Zhang LJ, et al. Antimicrobial peptide LL37 and MAVS signaling drive interferon- $\beta$ production by epidermal keratinocytes during skin injury. Immunity. 2016;45(1):119-130.

42. Oksala O, et al. Expression of proteoglycans and hyaluronan during wound healing. J Histochem Cytochem. 1995;43(2):125-135.

43. Taylor KR, et al. Recognition of hyaluronan released in sterile injury involves a unique receptor complex dependent on Toll-like receptor 4, CD44, and MD-2. J Biol Chem. 2007;282(25):18265-18275.

44. Gerdin B, Hällgren R. Localisation of hyaluronan in the human intestinal wall. Gut. 1991;32(7):760-762.

45. Dorschner RA, et al. Cutaneous injury induces the release of cathelicidin anti-microbial peptides active against group A Streptococcus. J Invest Dermatol. 2001;117(1):91-97.

46. Tenover FC, Goering RV. Methicillin-resistant Staphylococcus aureus strain USA300: origin and epidemiology. J Antimicrob Chemother. 2009;64(3):441-446.

47. Nizet V, et al. Innate antimicrobial peptide protects the skin from invasive bacterial infection. Nature. 2001;414(6862):454-457

48. Wirtz S, Neufert C, Weigmann B, Neurath MF. Chemically induced mouse models of intestinal inflammation. Nat Protoc. 2007;2(3):541-546.

49. Cooper HS, Murthy SN, Shah RS, Sedergran DJ. Clinicopathologic study of dextran sulfate sodium experimental murine colitis. Lab Invest. 1993;69(2):238-249.

50. Sasaki M, et al. Reversal of experimental colitis disease activity in mice following administration of an adenoviral IL-10 vector. J Inflamm (Lond). 2005;2(1):13.

51. Park YH, et al. Adequate dextran sodium sulfate-induced colitis model in mice and effective outcome measurement method. J Cancer Prev. 2015;20(4):260-267.

52. Orellana EA, Kasinski AL. Sulforhodamine B (SRB) Assay in Cell Culture to Investigate Cell Proliferation. Bio Protoc 2016;6(21):e1984.

53. Morioka Y, Yamasaki K, Leung D, Gallo RL. Cathelicidin antimicrobial peptides inhibit hyaluronan-induced cytokine release and modulate chronic allergic dermatitis. J Immunol. 2008;181(6):3915-3922.

54. Tripathi S, et al. Meta- and orthogonal integration of influenza "OMICs" data defines a role for UBR4 in virus budding. Cell Host Microbe. 2015;18(6):723-735.

55. Lee HG, Cowman MK. An agarose gel electrophoretic method for analysis of hyaluronan molecular weight distribution. Anal Biochem. 1994;219(2):278-287. 